\title{
The Relative Efficiency of the Conditional Root Square Estimation of Parameter in Inhomogeneous Equality Restricted Linear Model
}

\author{
Xiuli Nong \\ Guangxi Normal University for Nationalities, Chongzuo, China \\ Email: nxl1971@sina.com
}

Received May 22, 2012; revised June 30, 2012; accepted July 11, 2012

\begin{abstract}
This paper made a discuss on the relative efficiency of the generalized conditional root square estimation and the specific conditional root square estimation in paper $[1,2]$ in inhomogeneous equality restricted linear model. It is shown that the generalized conditional root squares estimation has not smaller the relative efficiency than the specific conditional root square estimation, by a constraint condition in root squares parameter, we compare bounds of them, thus, choose appropriate squares parameter, the generalized conditional root square estimation has the good performance on mean squares error.
\end{abstract}

Keywords: Generalized Conditional Root Square Estimation; Specific Conditional Root Square Estimation; Relative Efficiency

Consider inhomogeneous equality restricted linear model

$$
\left\{\begin{array}{l}
Y=X \beta+e, \quad E(e)=0, \quad \operatorname{Cov}(e)=\sigma^{2} I_{n} \\
R \beta=r
\end{array}\right.
$$

where $Y$ is a $n$-dimension vector, $X$ is a $n \times p$-order design matrix which is known, $e$ is $n \times 1$-random error vector, $I_{n}$ is $n$-order unit matrix, $\sigma^{2}>0$ is error variance, $R$ is $q \times p$-matrix, $r$ is $q \times 1$ dimension vector, $\beta \in B \hat{=}\{\beta: R \beta=r\}$ is a $n$-dimension parameter vector which is unknown. This paper all assume $X$ is full rank of column, $R$ is full rank of row.

The RLSE of the regression coefficient $\beta$ in the model (1) is noted as $\widetilde{\beta}=W X^{\prime} Y+V$ in the paper [1], where $W=S^{-1}-S^{-1} R^{\prime}\left[R S^{-1} R^{\prime}\right]^{-1} R S^{-1}, S=X^{\prime} X$,

$V=S^{-1} R^{\prime}\left[R S^{-1} R^{\prime}\right]^{-1} r$. In the paper [1], the conditional root square estimation of parameter of restricted linear model is derived, when multicollinearity of explanatory variables exists. It is shown that it has smaller mean squares error than the RLSE, and the admissibility of the conditional root estimation is discussed. Under the MDE matrix comparisons criterion, the necessary and sufficient condition or sufficient condition, under which CRSE is superior to RLSE, is obtained. Two methods (Root Trace, Variance Inflation Factor) are used to evaluate the optimal value. In the paper [2], proposes generalized root squares estimation in inhomogeneous
Equality Restricted Linear Model, we show that it have smaller mean squares error than the conditional root squares Estimation, and give display solution of generalize root squares estimation, propose the estimate methods of the optimal parameter value. Based on all the research work above, we made a discuss on the generalized conditional root square estimation and the specific conditional root square estimation in paper $[1,2]$ that the relative efficiency has in inhomogeneous equality restricted linear model. It is shown that the generalized conditional root squares estimation has not smaller the relative efficiency than the specific conditional root square estimation, by a constraint condition in root squares parameter, we compare bounds of them, thus, choose appropriate squares parameter, the generalized conditional root square estimation has good nature on terms mean squares error.

\section{Definition and Lemma}

Definition 1 [1] In the model (1), defined as $\tilde{\beta}(k)$ is the specific conditional root square estimation of $\beta$ :

$$
\tilde{\beta}(k)=\left(W^{+}\right)^{k} \tilde{\beta}=\left(W^{+}\right)^{k}\left(W X^{\prime} Y+V\right),
$$

where $0<k<1,\left(W^{+}\right)^{k}=Q \operatorname{diag}\left(\lambda_{1}^{-k}, \cdots, \lambda_{p-q}^{-k}, 0, \cdots, 0\right) Q^{\prime}$, $W, V$ defined as above paper, $Q$ is $p$-orthogonal matrix, make $Q^{\prime} W Q=\operatorname{diag}\left(\lambda_{1}, \lambda_{2}, \cdots, \lambda_{p-q}, 0, \cdots, 0\right) \hat{=} \Lambda, \quad \lambda_{i}$ is Non-zero characteristic values of $W$, and $\lambda_{1} \geq \lambda_{2} \geq \cdots \geq \lambda_{p-q}>0$. 
Definition 2 [2] In the model (1), defined as $\tilde{\beta}(K)$ is the generalized conditional root square estimation of $\beta$ :

$$
\tilde{\beta}(K)=\left(W^{+}\right)^{K} \tilde{\beta}=\left(W^{+}\right)^{K}\left(W X^{\prime} Y+V\right) .
$$

where $K=\operatorname{diag}\left(k_{1}, k_{2}, \cdots, k_{p}\right)$,

$$
\left(W^{+}\right)^{K}=Q \operatorname{diag}\left(\lambda_{1}^{-k_{1}}, \lambda_{2}^{-k_{2}} \cdots, \lambda_{p-q}^{-k_{p-q}}, 0, \cdots, 0\right) Q^{\prime} .
$$

said $k_{1}, k_{2}, \cdots, k_{p-q}$ is $p-q$-root square parameter, $W$, $Q, V$ defined as above paper.

Definition 3 [3] Two estimation $\tilde{\beta}$ and $\hat{\beta}$ of the model (1), defined as $e(\tilde{\beta}, \hat{\beta})=1-\frac{\operatorname{MSE}(\tilde{\beta})}{\operatorname{MSE}(\hat{\beta})}$ is elative efficiency of estimation $\tilde{\beta}$ for elative efficiency estimation of $\hat{\beta}$. If $\hat{\beta}$ is the best linear unbiased estimation of $\beta$, then note $e(\tilde{\beta}, \hat{\beta})=e(\tilde{\beta})$.

For the above definition 3 , if $0<e(\tilde{\beta}, \hat{\beta}) \leq 1$, then shows that $\tilde{\beta}$ is better than $\hat{\beta}$ under mean squares error and if the bigger of $e(\tilde{\beta}, \hat{\beta})$ (that efficiency highter), $\tilde{\beta}$ improve the degree of $\tilde{\beta}$ bigger.

Lemma 1 [1] $W S W=W, W S W^{\prime}=W$.

Lemma 2 [1] $W=S^{-1}-S^{-1} R^{\prime}\left[R S^{-1} R^{\prime}\right]^{-1} R S^{-1}$ is positive semidefinite matrix, and rank of $W$ is $p-q$.

Lemma 3 [1] Exist $Q$ is $p$-order orthogonal matrix, make $Q^{\prime} W Q=\operatorname{diag}\left(\lambda_{1}, \lambda_{2}, \cdots, \lambda_{p-q}, 0, \cdots, 0\right) \hat{=} \Lambda, \quad \lambda_{i}$ is Non-zero characteristic values of $W$, and $\lambda_{1} \geq \lambda_{2} \geq \cdots \geq \lambda_{p-q}>0$.

Lemma 4 [1] Mean squares error of $\tilde{\beta}$ is $\operatorname{MSE}(\tilde{\beta})=\sigma^{2} \sum_{i=1}^{p-q} \lambda_{i}, \quad \lambda_{i}$ is Non-zero characteristic values of $W$, and $\lambda_{1} \geq \lambda_{2} \geq \cdots \geq \lambda_{p-q}>0$.

Lemma 5 [1] Assume $Q^{\prime} V=\left(b_{1}, b_{2}, \cdots, b_{p-q}, \cdots, b_{p}\right)^{\prime}$ then

$$
\begin{aligned}
Q^{\prime} \beta & =\alpha+Q^{\prime} V \\
& =\left(\alpha_{1}+b_{1}, \cdots, \alpha_{p-q}+b_{p-q}, \cdots, \alpha_{p}+b_{p}\right)^{\prime},
\end{aligned}
$$

where $\alpha_{p-q+1}=\alpha_{p-q+2}=\cdots=\alpha_{p}=0$.

\section{Main Results}

We can prove the following exist theorem $0<e(\tilde{\beta}(k)) \leq e(\tilde{\beta}(K)) \leq 1$ and bound of $e(\tilde{\beta}(k))$ and $e(\tilde{\beta}(K))$. Now, we have the following lemma.

Assume $\alpha=Q^{\prime}(\beta-V)=\left(\alpha_{1}, \alpha_{2}, \cdots, \alpha_{p}\right)^{\prime}$, then $\alpha_{p-q+1}=\alpha_{p-q+2}=\cdots=\alpha_{p}=0$.

And the RLSE of $\alpha$ is

$$
\begin{aligned}
\tilde{\alpha} & =Q^{\prime}(\tilde{\beta}-V)=Q^{\prime}\left(W X^{\prime} Y+V-V\right)=Q^{\prime} W X^{\prime} Y \\
& =Q^{\prime} W Q Q^{\prime} X^{\prime} Y=\Lambda Q^{\prime} X^{\prime} Y
\end{aligned}
$$

accordingly, the specific conditional root square estimation of $\alpha$ is

$$
\begin{aligned}
\tilde{\alpha}(k) & =Q^{\prime}(\tilde{\beta}(k)-V)=Q^{\prime}\left[\left(W^{+}\right)^{k}\left(W X^{\prime} Y+V\right)-V\right] \\
& =Q^{\prime}\left(W^{+}\right)^{k} W X^{\prime} Y+Q^{\prime}\left(W^{+}\right)^{k} V-Q^{\prime} V \\
& =Q^{\prime}\left(W^{+}\right)^{k} Q Q^{\prime} W Q Q^{\prime} X^{\prime} Y+Q^{\prime}\left(W^{+}\right)^{k} Q Q^{\prime} V-Q^{\prime} V \\
& =\left(\Lambda^{+}\right)^{k} \Lambda Q^{\prime} X^{\prime} Y+\left(\Lambda^{+}\right)^{k} Q^{\prime} V-Q^{\prime} V \\
& =\left(\Lambda^{+}\right)^{k} \tilde{\alpha}+\left[\left(\Lambda^{+}\right)^{k}-I\right] Q^{\prime} V, \\
& 0<k<1 .
\end{aligned}
$$

similarly, the generalized conditional root square estimation of $\alpha$ is

$$
\begin{gathered}
\tilde{\alpha}(K)=Q^{\prime}(\tilde{\beta}(K)-V)=\left(\Lambda^{+}\right)^{K} \tilde{\alpha}+\left[\left(\Lambda^{+}\right)^{K}-I\right] Q^{\prime} V, \\
K=\operatorname{diag}\left(k_{1}, k_{2}, \cdots, k_{p}\right) .
\end{gathered}
$$

Lemma $6 \operatorname{MSE}(\tilde{\alpha}(K))=\sum_{i=1}^{p-q} g\left(k_{i}\right)$, where

$g\left(k_{i}\right)=\sigma^{2} \lambda_{i}^{1-2 k_{i}}+\left(\alpha_{i}+b_{i}\right)^{2}\left(\lambda_{i}^{-k_{i}}-1\right)^{2}$.

Proof:

when $k_{i}=0, \sum_{i=1}^{p-q} g(0)=\sigma^{2} \sum_{i=1}^{p-q} \lambda_{i}=\operatorname{MSE}(\tilde{\beta})$.

Because $\tilde{\alpha}(K)=\left(\Lambda^{+}\right)^{K} \tilde{\alpha}+\left[\left(\Lambda^{+}\right)^{K}-I\right] Q^{\prime} V$, so

$\operatorname{MSE}(\tilde{\alpha}(K))=\operatorname{tr}(\operatorname{cov}(\tilde{\alpha}(K)))+\|E(\tilde{\alpha}(K))-\alpha\|^{2}$.

Because

$$
\begin{aligned}
& \operatorname{tr}(\operatorname{cov}(\tilde{\alpha}(K))) \\
& =\operatorname{tr}\left(\operatorname{cov}\left[\left(\Lambda^{+}\right)^{K} \tilde{\alpha}+\left(\left(\Lambda^{+}\right)^{K}-I\right) Q^{\prime} V\right]\right. \\
& =\operatorname{tr}\left(\operatorname{cov}\left[\left(\Lambda^{+}\right)^{K} \tilde{\alpha}\right]=\operatorname{tr}\left(\Lambda^{+}\right)^{K} \operatorname{cov}(\tilde{\alpha})\left(\Lambda^{+}\right)^{K}\right. \\
& =\operatorname{tr}\left(\Lambda^{+}\right)^{K} \sigma^{2} W\left(\Lambda^{+}\right)^{K}=\sum_{i=1}^{p-q} \sigma^{2} \lambda_{i}^{1-2 k_{i}}
\end{aligned}
$$

So

$$
\begin{aligned}
& E(\tilde{\alpha}(K))=E\left[\left(\Lambda^{+}\right)^{k} \tilde{\alpha}+\left[\left(\Lambda^{+}\right)^{k}-I\right] Q^{\prime} V\right] \\
& =\left(\Lambda^{+}\right)^{k} \alpha+\left[\left(\Lambda^{+}\right)^{k}-I\right] Q^{\prime} V\|E(\tilde{\alpha}(K))-\alpha\|^{2} \\
& =\left\|\left(\Lambda^{+}\right)^{k} \alpha+\left[\left(\Lambda^{+}\right)^{k}-I\right] Q^{\prime} V-\alpha\right\|^{2} \\
& =\sum_{i=1}^{p-q}\left(\alpha_{i}+b_{i}\right)^{2}\left(\lambda_{i}^{-k_{i}}-1\right)^{2}
\end{aligned}
$$


Lemma $7 \operatorname{MSE}(\tilde{\beta}(k))=\operatorname{MSE}(\tilde{\alpha}(k))$,

$\operatorname{MSE}(\tilde{\beta}(K))=\operatorname{MSE}(\tilde{\alpha}(K))$.

Lemma 8 When $0 \leq k \leq \frac{1}{2}$, exist $k_{0}\left(0<k_{0} \leq \frac{1}{2}\right)$, when $0<k<k_{0}$, then

$$
g(k)=\sigma^{2} \lambda^{1-2 k}+(\alpha+b)^{2}\left(\lambda^{-k}-1\right)^{2}
$$

has minimum value.

Proof: Note $g_{1}(k)=\sigma^{2} \lambda^{1-2 k}$,

$g_{2}(k)=-(\alpha+b)^{2}\left(\lambda^{-k}-1\right)^{2}$, then $g(k)=g_{1}(k)+g_{2}(k)$.

For $g_{1}(k)$, we have $g_{1}^{\prime}(k)=-2 \sigma^{2} \lambda^{1-2 k} \ln \lambda$. When $0 \leq k \leq \frac{1}{2}$, if $\lambda \geq 1$, then $\ln \lambda \geq 0, \lambda^{1-2 k} \geq 1$; if $0<\lambda \leq 1$, then $\ln \lambda \leq 0, \lambda^{1-2 k} \leq 1$. When $\lambda \geq 1, g_{1}^{\prime}(k)<0$. So $g_{1}(k)$ is a monotonically decreasing function in $\left(0, \frac{1}{2}\right)$.

For $g_{2}(k)$, when $\lambda \geq 1$, we have $g_{2}^{\prime}(k)=-2(\alpha+b)^{2}\left(\lambda^{-k}-1\right) \lambda^{-k} \ln \lambda \geq 0$. This means

$g_{2}(k)$ is a monotonically increasing function in $\left(0, \frac{1}{2}\right)$. So, there always exist $k_{0}\left(0<k_{0} \leq \frac{1}{2}\right)$, when $0<k<k_{0}$, we have $g^{\prime}(k)=g_{1}^{\prime}(k)+g_{2}^{\prime}(k)<0$, so $g(k)$ is a monotonically decreasing function in $\left(0, k_{0}\right), g(k)<$ $g(0), g(k)$ has minimum value.

Lemma 9 In the model (1), for

$g(k)=\sigma^{2} \lambda^{1-2 k}+(\alpha+b)^{2}\left(\lambda^{-k}-1\right)^{2}$, when

$k=\frac{\ln \frac{(\alpha+b)^{2}+\sigma^{2} \lambda}{(\alpha+b)^{2}}}{\ln \lambda}$, then $g(k)$ has minimum value.

Proof: According to lemma 8,

$$
\begin{aligned}
g^{\prime}(k) & =g_{1}^{\prime}(k)+g_{2}^{\prime}(k) \\
& =-2 \sigma^{2} \lambda^{1-2 k} \ln \lambda-2(\alpha+b)^{2}\left(\lambda^{-k}-1\right) \lambda^{-k} \ln \lambda .
\end{aligned}
$$

Let $g^{\prime}(k)=0$, we get

$$
-2 \sigma^{2} \lambda^{1-2 k} \ln \lambda-2(\alpha+b)^{2}\left(\lambda^{-k}-1\right) \lambda^{-k} \ln \lambda=0,
$$

when $\lambda=1$, the solution of this equation is $k=0$; when $\lambda \neq 1$, the solution of this equation is

$\lambda^{-k}=\frac{(\alpha+b)^{2}}{\sigma^{2} \lambda+(\alpha+b)^{2}}$, that $k=\frac{\ln \frac{(\alpha+b)^{2}+\sigma^{2} \lambda}{(\alpha+b)^{2}}}{\ln \lambda}$.
So $k=\left\{\begin{array}{cc}0, & \lambda=1 \\ \frac{\ln \frac{(\alpha+b)^{2}+\sigma^{2} \lambda}{(\alpha+b)^{2}}}{\ln \lambda}, & \lambda \neq 1\end{array}\right.$. Therefore when $k=\frac{\ln \frac{(\alpha+b)^{2}+\sigma^{2} \lambda}{(\alpha+b)^{2}}}{\ln \lambda}$, then $g(k)$ has minimum value.

Lemma 10 In the model (1), exist root square parameter $0<k<1$, then mean squares error of $\tilde{\beta}(k)$ is

$$
\operatorname{MSE}(\tilde{\beta}(k))=\sum_{i=1}^{p-q}\left[\sigma^{2} \lambda_{i}^{1-2 k}+\left(\alpha_{i}+b_{i}\right)^{2}\left(\lambda_{i}^{-k}-1\right)^{2}\right] .
$$

Lemma 11 In the model (1), $\prod_{i=1}^{p-q} \lambda_{i}>1$, always exist $0 \leq k \leq \frac{1}{2}$, then $\operatorname{MSE}(\tilde{\beta}(k)) \leq \operatorname{MSE}(\tilde{\beta})$.

Proof: Based on the lemma 9 and lemma 10.

Theorem 1 In the model (1), $\prod_{i=1}^{p-q} \lambda_{i}>1$, always exist $0 \leq k \leq \frac{1}{2}$, then $0<e(\tilde{\beta}(k)) \leq 1$.

Proof: Based lemma 11 and definition 3, we get the conclusion.

Theorem 2 In the model (1), for $k>0$, exist $K=\operatorname{diag}\left(k_{1}, \cdots, k_{p}\right)$, then $0 \leq e(\tilde{\beta}(K), \tilde{\beta}(k)) \leq 1$.

Proof: For $k>0$, if $k=\frac{\ln \frac{\left(\alpha_{i}+b_{i}\right)^{2}+\sigma^{2} \lambda}{\left(\alpha_{i}+b_{i}\right)^{2}}}{\ln \lambda}$, $i=1,2, \cdots, p$, choose $k_{i}=k$, we have $e(\tilde{\beta}(K), \tilde{\beta}(k))=0$.

Assume at least exist $i$, that $k \neq \frac{\ln \frac{\left(\alpha_{i}+b_{i}\right)^{2}+\sigma^{2} \lambda}{\left(\alpha_{i}+b_{i}\right)^{2}}}{\ln \lambda}$, assume $k_{1}=\frac{\ln \frac{\left(\alpha_{1}+b_{1}\right)^{2}+\sigma^{2} \lambda}{\left(\alpha_{1}+b_{1}\right)^{2}}}{\ln \lambda}$, where $k_{i}=k$, $i=2,3, \cdots, p$, based on lemma 6 and, we have

$$
\begin{aligned}
& \operatorname{MSE}(\tilde{\beta}(k))-\operatorname{MSE}(\tilde{\beta}(K)) \\
& =\sum_{i=1}^{p-q} g(k)-\sum_{i=1}^{p-q} g_{i}\left(k_{i}\right)=g(k)-g_{1}\left(k_{1}\right),
\end{aligned}
$$

based on lemma 9, we have $g(k)-g_{1}\left(k_{1}\right)>0$, then $\operatorname{MSE}(\tilde{\beta}(k))-\operatorname{MSE}(\tilde{\beta}(K))>0$, so

$e(\tilde{\beta}(K), \tilde{\beta}(k))>0$. 
For above theorem, then $0 \leq e(\tilde{\beta}(K), \tilde{\beta}(k)) \leq 1$.

Using theorem 2, we get the following the conclusion.

Inference 1 In the model (1), for $k>0$, exist $K=\operatorname{diag}\left(k_{1}, \cdots, k_{p}\right)$, then $0<e(\tilde{\beta}(k)) \leq e(\tilde{\beta}(K)) \leq 1$.

Inference 2 In the model (1), if $\beta_{i}(i=1,2, \cdots, p)$ Are not all equal, then $0<e(\tilde{\beta}(k)) \leq e(\tilde{\beta}(K)) \leq 1$.

Proof: Because $\alpha=Q^{\prime}(\beta-V), Q$ is orthogonal matrix, so when $\beta_{1} \neq \beta_{2}$, then $\alpha_{1} \neq \alpha_{2}$, based on theorem 2 , we get the conclusion.

Theorem 3 In the model (1), when

$$
0<k \leq \frac{\frac{\ln \left[\left(\alpha_{p-q}+b_{p-q}\right)^{2}+\sigma^{2} \lambda_{p-q}\right]}{\left(\alpha_{p-q}+b_{p-q}\right)^{2}}}{\ln \lambda_{1}},
$$

then $e(\tilde{\beta}(k)) \geq e_{0}>0$, where

$$
e_{0}=\frac{\sigma^{2} \lambda_{p-q}-\sigma^{2} \lambda_{1}^{1-2 k}+\left(\alpha_{p-q}+b_{p-q}\right)^{2}\left(\lambda_{p-q}^{-k}-1\right)^{2}}{\sigma^{2} \lambda_{1}},
$$

$\alpha_{p-q}$ is $\alpha=Q^{\prime}(\beta-V)$ the largest component of module.

Proof: Assume $\sigma^{2} \lambda_{1} \geq\left(\alpha_{p-q}+b_{p-q}\right)^{2}$, then

$$
\begin{aligned}
& \operatorname{MSE} \tilde{\beta}-\operatorname{MSE}(\tilde{\beta}(k)) \\
& =\sigma^{2} \sum_{i=1}^{p-q} \lambda_{i}-\sum_{i=1}^{p-q}\left[\sigma^{2} \lambda_{i}^{1-2 k}+\left(\alpha_{i}+b_{i}\right)^{2}\left(\lambda_{i}^{-k}-1\right)^{2}\right] \\
& =\sigma^{2} \sum_{i=1}^{p-q}\left[\lambda_{i}-\lambda_{i}^{1-2 k}-\frac{\left(\alpha_{i}+b_{i}\right)^{2}\left(\lambda_{i}^{-k}-1\right)^{2}}{\sigma^{2}}\right] \\
& =\sigma^{2} \sum_{i=1}^{p-q} \lambda_{i}\left[1-\lambda_{i}^{-2 k}-\frac{\left(\alpha_{i}+b_{i}\right)^{2}\left(\lambda_{i}^{-k}-1\right)^{2}}{\sigma^{2} \lambda_{i}}\right] .
\end{aligned}
$$

Assume

$$
e_{0}=\frac{\sigma^{2} \lambda_{p-q}-\sigma^{2} \lambda_{1}^{1-2 k}-\left(\alpha_{p-q}+b_{p-q}\right)^{2}\left(\lambda_{p-q}^{-k}-1\right)^{2}}{\sigma^{2} \lambda_{1}},
$$

then

$$
\operatorname{MSE} \tilde{\beta}-\operatorname{MSE}(\tilde{\beta}(k)) \geq \sigma^{2} \sum_{i=1}^{p-q} \lambda_{i} e_{0}=e_{0} \operatorname{MSE} \tilde{\beta},
$$

so $e(\tilde{\beta}(k))=1-\frac{\operatorname{MSE}(\tilde{\beta}(k))}{\operatorname{MSE}(\tilde{\beta})} \geq e_{0}>0$.

Theorem 4 In the model (1), for $K=\operatorname{diag}\left(k_{1}, \cdots, k_{p}\right)$,

if $k_{i}=\frac{\ln \frac{\left(\alpha_{i}+b_{i}\right)^{2}+\sigma^{2} \lambda}{\left(\alpha_{i}+b_{i}\right)^{2}}}{\ln \lambda}\left(\right.$ or $\left.\lambda^{-k_{i}} \frac{\left(\alpha_{i}+b_{i}\right)^{2}}{\sigma^{2} \lambda_{i}+\left(\alpha_{i}+b_{i}\right)^{2}}\right)$, $i=1,2, \cdots, p$, then $e(\tilde{\beta}(K)) \geq e_{1}>0$, Where

$$
e_{1}=\frac{\sigma^{2}}{\sigma^{2} \lambda_{1}+\left(\alpha_{p-q}+b_{p-q}\right)^{2}} \text {. }
$$

Proof:

$$
\begin{aligned}
\operatorname{MSE} \tilde{\beta}-\operatorname{MSE}(\tilde{\beta}(K)) \\
=\sigma^{2} \sum_{i=1}^{p-q} \lambda_{i}-\sum_{i=1}^{p-q}\left[\sigma^{2} \lambda_{i}^{1-2 k_{i}}+\left(\alpha_{i}+b_{i}\right)^{2}\left(\lambda_{i}^{-k_{i}}-1\right)^{2}\right] \\
=\sum_{i=1}^{p-q}\left[\sigma^{2} \lambda_{i}\left(1-\lambda_{i}^{-2 k_{i}}\right)-\left(\alpha_{i}+b_{i}\right)^{2}\left(\lambda_{i}^{-k_{i}}-1\right)^{2}\right] \\
=\sum_{i=1}^{p-q}\left[\sigma^{2} \lambda_{i}\left(1-\frac{\left(\alpha_{i}+b_{i}\right)^{4}}{\left[\sigma^{2} \lambda_{i}+\left(\alpha_{i}+b_{i}\right)^{2}\right]^{2}}\right]\right. \\
\left.=-\left(\alpha_{i}+b_{i}\right)^{2}\left(\frac{\left(\alpha_{i}+b_{i}\right)^{2}}{\sigma^{2} \lambda_{i}+\left(\alpha_{i}+b_{i}\right)^{2}}-1\right)\right] \\
=\sum_{i=1}^{p-q}\left[\sigma^{2} \lambda_{i} \frac{\sigma^{4} \lambda_{i}^{2}+2 \sigma^{2} \lambda_{i}\left(\alpha_{i}+b_{i}\right)^{2}}{\left[\sigma^{2} \lambda_{i}+\left(\alpha_{i}+b_{i}\right)^{2}\right]^{2}}-\frac{\left(\alpha_{i}+b_{i}\right)^{2} \sigma^{4} \lambda_{i}^{2}}{\left[\sigma^{2} \lambda_{i}+\left(\alpha_{i}+b_{i}\right)^{2}\right]^{2}}\right] \\
=\sum_{i=1}^{p-q} \frac{\sigma^{6} \lambda_{i}^{3}+\sigma^{4} \lambda_{i}^{2}\left(\alpha_{i}+b_{i}\right)^{2}}{\left[\sigma^{2} \lambda_{i}+\left(\alpha_{i}+b_{i}\right)^{2}\right]^{2}}=\sum_{i=1}^{p-q} \frac{\sigma^{4} \lambda_{i}}{\sigma^{2} \lambda_{i}+\left(\alpha_{i}+b_{i}\right)^{2}} \\
=\sum_{i=1}^{p-q} \sigma^{2} \lambda_{i} \frac{\sigma^{2}}{\sigma^{2} \lambda_{i}+\left(\alpha_{i}+b_{i}\right)^{2}} \\
\geq \frac{\sigma^{2}}{\sigma^{2} \lambda_{1}+\left(\alpha_{p-q}+b_{p-q}\right)^{2} \sum_{i=1}^{p-q} \sigma^{2} \lambda_{i}} \\
=\frac{\sigma^{2}}{\sigma^{2} \lambda_{1}+\left(\alpha_{p-q}+b_{p-q}\right)^{2}} \mathrm{MSE}_{\tilde{\beta}} .
\end{aligned}
$$

Therefore $e(\tilde{\beta}(K)) \geq \frac{\sigma^{2}}{\sigma^{2} \lambda_{1}+\left(\alpha_{p-q}+b_{p-q}\right)^{2}}>0$, let $e_{1}=\frac{\sigma^{2}}{\sigma^{2} \lambda_{1}+\left(\alpha_{p-q}+b_{p-q}\right)^{2}}$, then $e(\tilde{\beta}(K)) \geq e_{1}>0$.

Theorem 5 In the model (1), assume the non-zero characteristic root $\lambda_{i}$ of $W$ are not all equal $(i=1,2, \cdots, p)$, for the efficiency lower bound $e_{0}$ of $\tilde{\beta}(k)$ and the efficiency lower bound $e_{1}$ of $\tilde{\beta}(K)$, the relationship of them is $e_{0}<e_{1}$.

Proof: By theorems 3 and 4 , we get

$$
e_{0}=\frac{\sigma^{2} \lambda_{p-q}-\sigma^{2} \lambda_{1}^{1-2 k}-\left(\alpha_{p-q}+b_{p-q}\right)^{2}\left(\lambda_{p-q}^{-k}-1\right)^{2}}{\sigma^{2} \lambda_{1}},
$$




$$
e_{1}=\frac{\sigma^{2}}{\sigma^{2} \lambda_{1}+\left(\alpha_{p-q}+b_{p-q}\right)^{2}},
$$

note $\frac{e_{1}}{e_{0}}=\frac{D_{1}}{D_{2}}$ then

$$
\begin{aligned}
D_{1}= & \sigma^{4} \lambda_{1}, \\
D_{2}= & \sigma^{4} \lambda_{1} \lambda_{p-q}-\sigma^{4} \lambda_{1}^{2-2 k}-\sigma^{2} \lambda_{1}\left(\alpha_{p-q}+b_{p-q}\right)^{2}\left(\lambda_{p-q}^{-k}-1\right)^{2} \\
& +\sigma^{2} \lambda_{p-q}\left(\alpha_{p-q}+b_{p-q}\right)^{2}-\sigma^{2} \lambda_{1}^{1-2 k}\left(\alpha_{p-q}+b_{p-q}\right)^{2} \\
& -\left(\alpha_{p-q}+b_{p-q}\right)^{4}\left(\lambda_{p-q}^{-k}-1\right)^{2}
\end{aligned}
$$

Then

$$
\begin{aligned}
& D_{1}-D_{2} \\
&=\sigma^{4} \lambda_{1}-\sigma^{4} \lambda_{1} \lambda_{p-q}+\sigma^{4} \lambda_{1}^{2-2 k} \\
&+\sigma^{2} \lambda_{1}\left(\alpha_{p-q}+b_{p-q}\right)^{2}\left(\lambda_{p-q}^{-k}-1\right)^{2} \\
&-\sigma^{2} \lambda_{p-q}\left(\alpha_{p-q}+b_{p-q}\right)^{2}+\sigma^{2} \lambda_{1}^{1-2 k}\left(\alpha_{p-q}+b_{p-q}\right)^{2} \\
&+\left(\alpha_{p-q}+b_{p-q}\right)^{4}\left(\lambda_{p-q}^{-k}-1\right)^{2} \\
&= \sigma^{4} \lambda_{1}+\sigma^{2} \lambda_{1}^{1-2 k}\left[\sigma^{2} \lambda_{1}+\left(\alpha_{p-q}+b_{p-q}\right)^{2}\right] \\
&-\sigma^{2} \lambda_{p-q}\left[\sigma^{2} \lambda_{1}+\left(\alpha_{p-q}+b_{p-q}\right)^{2}\right] \\
&+\left(\alpha_{p-q}+b_{p-q}\right)^{2}\left(\lambda_{p-q}^{-k}-1\right)^{2}\left[\sigma^{2} \lambda_{1}+\left(\alpha_{p-q}+b_{p-q}\right)^{2}\right] \\
&= \sigma^{4} \lambda_{1}+\left[\sigma^{2} \lambda_{1}+\left(\alpha_{p-q}+b_{p-q}\right)^{2}\right] \\
& \cdot\left[\sigma^{2}\left(\lambda_{1}^{1-2 k}-\lambda_{p-q}\right)+\left(\alpha_{p-q}+b_{p-q}\right)^{2}\left(\lambda_{p-q}^{-k}-1\right)^{2}\right] .
\end{aligned}
$$

As $\lambda_{i}$ are not all equal $(i=1,2, \cdots, p)$, therefore $\lambda_{1}>\lambda_{p-q}$, also $1-2 k>0$, then $\lambda_{1}^{1-2 k}-\lambda_{p-q}>0$, thus $D_{1}-D_{2}>0$.

That $e_{0}<e_{1}$.

\section{REFERENCES}

[1] X.-L. Nong and W.-R. Liu, “The Conditional Root Square Estimation of Parameter of Restricted Linear Model," Journal of Chongqing Normal University (Natural Science Edition), No. 2, 2007, pp. 24-28.

[2] X.-L. Nong, W.-R. Liu, et al., "The Generalized Conditional Root Squares Estimation of Parameter in Restricted Linear Model," Journal of Guangxi University of Technology, Vol. 18, No. 3, 2007, pp. 24-27.

[3] P.-H. Wang, "The Relative Efficiency of the Generalized Ridge Estimation,” Journal of QuanZhou Normal College, Vol. 2, 1998, pp. 13-15. 